\title{
SPECTROSCOPY, PHOTOMETRY AND MICRO-ARCSEC ASTROMETRY OF BINARIES WITH THE GAIA SPACE MISSION AND WITH THE RAVE EXPERIMENT
}

\author{
Tomaž Zwitter ${ }^{1}$ and Ulisse Munari ${ }^{2}$
}

\section{RESUMEN}

La misión astrométrica GAIA de la ESA va a ser muy eficiente para descubrir estrellas dobles y múltiples de cualquier período, desde minutos hasta millones de años. Se presentan los parámetros principales del diseño revisado de la misión. Se estima la fracción de binarias que serán descubiertas mediante astrometría, fotometría y espectroscopía a bordo. Finalmente, se resumen las observaciones que confirman la posibilidad de medir parámetros como las masas, los radios y las distancias espectroscópicas exclusivamente a partir de datos de GAIA. GAIA no volará sino hasta 2010, pero el experimento de velocidades radiales (RAVE) ya comenzó este año. Mostramos que las observaciones espectroscópicas de éste son capaces de descubrir una gran parte de los sistemas binarios aún desconocidos.

The GAIA astrometric mission of ESA will be very efficient in discovering binary and multiple stars with any orbital period, from minutes to millions of years. The main parameters of the revised mission design are presented. Next we estimate the fraction of binary stars discovered by means of astrometry, photometry and on-board spectroscopy. Finally we summarize observations that confirm the ability to measure physical parameters like masses, radii and spectroscopic distance from GAIA data alone. GAIA will fly only in 2010, but the radial velocity experiment (RAVE) has started this year. We show that its spectroscopic observations have the capacity to discover a large fraction of so far unknown binary systems.

\section{Key Words: BINARIES: ECLIPSING - BINARIES: SPECTROSCOPIC - STARS: FUNDAMENTAL PARAMETERS - SURVEYS: (GAIA, RAVE)}

\section{INTRODUCTION}

The Hipparcos astrometric mission of ESA in the early nineties had a remarkable success. For 118,218 targets listed in the Hipparcos catalogue it was able to obtain astrometry of good precision (10\% error at $100 \mathrm{pc}$ ), accurate proper motions (error of $1 \mathrm{mas} / \mathrm{yr}$ ) and three-colour photometry. The satellite scanned the sky, each object was observed in $\sim 30$ independent geometrical observations, so a simple fiveparameter model could be expanded to discover a number of new double and multiple stars. The actual number of observations of each target was even larger $(\sim 110)$ so a number of variable stars as well as eclipsing binaries were discovered from Hipparcos light curves (ESA SP-402, vol. 12).

All these results prompted preparations for its successor even before the Hipparcos observations were completed. Already in 1995 ESA embraced the idea of the GAIA mission (ESA SP-379). It was

\footnotetext{
${ }^{1}$ University of Ljubljana, Department of Physics, Ljubljana, Slovenia. email: tomaz.zwitter@fmf.uni-lj.si

${ }^{2}$ Osservatorio Astronomico di Padova, Sede di Asiago, Asiago (VI), Italy. email: munari@pd.astro.it
}

later approved as a Cornerstone 6 mission and in May 2002 it was re-approved within the new Cosmic Vision 2020 programme of ESA to fly around 2010. GAIA will improve the results of Hipparcos in many areas (see Table 1). Its astrometry will be of a much higher precision and reaching for fainter targets. Astrometry will be used to obtain 5 coordinates in space and velocity of each object, with an on-board spectroscopy supplying the missing radial velocity. Accurate multi-band photometry will be used to assess chromaticity corrections and to judge basic parameters of stellar atmospheres like temperature, gravity and metallicity. The plan is to observe any of the $\sim$ billion stars brighter than $V=20$ repeatedly when the scanning satellite will bring it into the field of view. This is very different from high-precision but pointed observations of a moderate number of targets to be observed by the SIM mission. Some smaller photometric missions (COROT, Eddington) are discussed elsewhere in this volume (Maceroni 2004).

Scientific drivers and technical solutions of the GAIA mission have been extensively described in 
TABLE 1

SUMMARY OF CAPABILITIES OF HIPPARCOS, SIM AND GAIA MISSIONS ${ }^{a}$

\begin{tabular}{|c|c|c|c|}
\hline & Hipparcos & SIM & GAIA \\
\hline agency & ESA & NASA & ESA \\
\hline mission lifetime & 4 yrs & 5 yrs & $5 \mathrm{yrs}$ \\
\hline launch & 1989 & end of 2009 & 2010 \\
\hline No. of stars & 120,000 & 10,000 & 1 billion \\
\hline mag. limit & 12 & 20 & 20 \\
\hline astrometric accuracy & 1 mas $($ at $V=10)$ & $3 \mu$ as $($ at $\mathrm{V}=20$ ) & $\begin{array}{l}3 \mu \text { as }(\text { at } V=12) \\
10 \mu \text { as }(\text { at } V=15) \\
200 \mu \text { as }(\text { at } V=20)\end{array}$ \\
\hline photometric filters & 3 (BBP) & & $5\left(\mathrm{BBP}^{\mathrm{b}}\right)$, up to $16\left(\mathrm{MBP}^{\mathrm{c}}\right)$ \\
\hline radial velocity & not available & not available & $\begin{array}{l}\sigma \sim 1 \mathrm{~km} \mathrm{~s}^{-1}\left(\text { at } \mathrm{I}_{\mathrm{C}}=14\right) \\
\sigma \sim 10 \mathrm{~km} \mathrm{~s}^{-1}\left(\text { at } \mathrm{I}_{\mathrm{C}}=16\right)\end{array}$ \\
\hline epochs on each target & $\sim 110$ & pointed & $\begin{array}{l}\left.\sim 82 \text { (astrometry, } \mathrm{BBP}^{\mathrm{b}}\right) \\
\left.\sim 200 \text { (MBP }^{\mathrm{c}}\right) \\
\sim 100 \text { (spectroscopy) }\end{array}$ \\
\hline
\end{tabular}

${ }^{a}$ Adapted from ESA-SCI(2000)4, Jordi et al. (2003), and Munari et al. (2003).

${ }^{b}$ broad-band photometry.

cmedium-band photometry.

the ESA's Concept and Technology Study (ESASCI (2000)4) and in papers of Gilmore et al. (1998) and Perryman et al. (2001). Several conferences have been devoted to definition of its scientific goals (Straižys 1999, Bienaymé \& Turon 2002, Vansevičius, Kučinskas \& Sudžius 2002, and Munari 2003). Here we will start with a non-technical description of the scientific payload of the satellite and continue with assessment of GAIA's capabilities for discovery and modelling of binary and multiple stellar systems. In the end we will briefly describe the ground-based project RAVE which is about to begin collecting radial velocities and near-IR spectra for the brighter end of the GAIA objects within this year.

\section{GAIA ASTROMETRY}

GAIA astrometry builds on the concept proved by the Hipparcos satellite. Two astrometric telescopes are used. They are pointing in directions perpendicular to the telescope axis which are $106^{\circ}$ apart. The image of both telescopes forms in a common focal plane. The satellite rotates around its axis every 6 hours, so the stars drift along the focal plane. The plane is covered with an array of 110 astrometric CCDs read in a time-delayed-integration mode, so that angles along the drift direction between stars seen by the first and the second telescope can be measured with extreme accuracy. The satellite axis precesses in 70 days along the cone with a half opening angle of $50^{\circ}$ around the direction pointing away from the Sun. Combination of rotation, precession and motion around the Sun guarantees that each point in the sky is observed in many epochs during the 5 year mission lifetime.

Extreme astrometric precision requires very stable observing conditions. The whole structure will be made of $\mathrm{SiC}$, but even so the temperature variations could not exceed $20 \mu \mathrm{K}$ on a time-scale of a 6 -hour rotation period. This can be met in an orbit close to the second Lagrangian point of the Earth-Sun system. The satellite will be always kept out of the Earth shadow and the instruments will be shielded from direct illumination by a Sun shield.

Astrometric accuracy of $10 \mu$ as will be achieved for a $V=15$ mag star after combination of all observations during the $5 \mathrm{yr}$ mission. It corresponds to a $10 \%$ error in distance at $10 \mathrm{kpc}$. The proper motion error of $10 \mu \mathrm{as} \mathrm{yr}^{-1}$ equals to a $1 \mathrm{~km} \mathrm{~s}^{-1}$ error in transverse velocity at $20 \mathrm{kpc}$. So GAIA will be able to directly measure distances and transverse motions with remarkable accuracy for a representative sample of stars in the Galaxy.

\section{GAIA PHOTOMETRY}

Each star passing the field of view of an astrometric telescope will also be observed in 4 broad 


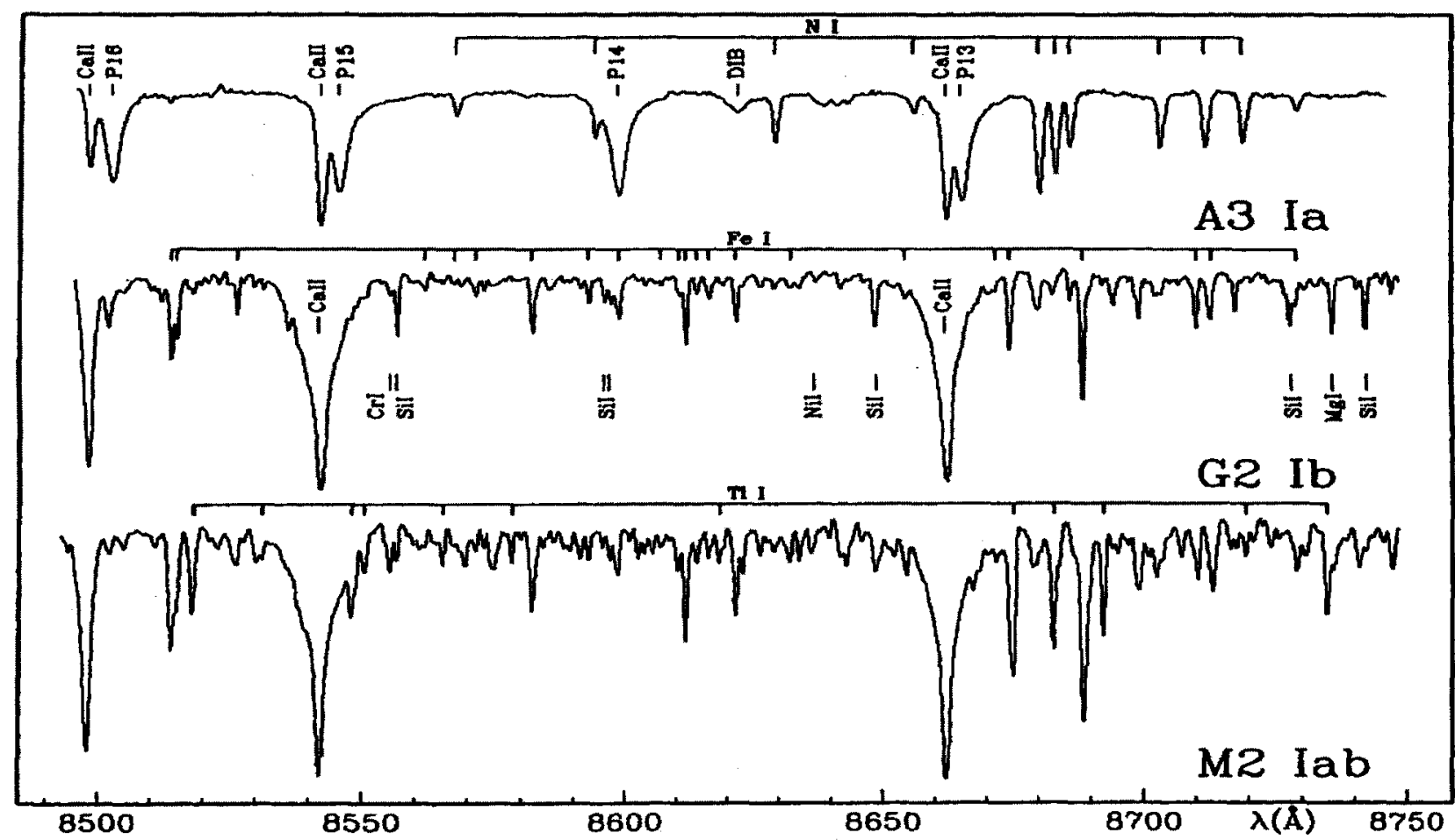

Fig. 2. Spectra of A, G and M supergiants in the GAIA spectral interval. From Munari (1999).

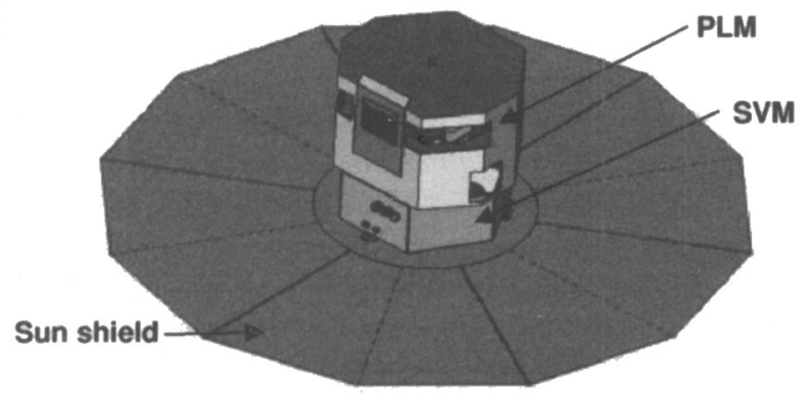

Fig. 1. GAIA design with payload module (PLM) and service module (SVM). The antenna pointing toward the Earth is on the other side of the Sun shield.

$(\Delta \lambda / \lambda \sim 0.25)$ photometric bands and in white light. Typical errors per observation will be between 0.01 and $0.02 \mathrm{mag}$ at $\mathrm{V}=18.5$. In addition the edges of the focal plane of the spectroscopic telescope contain 32 CCDs that will be used for mediumband photometry. Altogether up to 16 medium-band $(\Delta \lambda / \lambda \sim 0.1)$ filters will be used.

Broad band photometry will be excellent to measure the general energy distribution of the target. Medium-band filters are designed to maximize the astrophysical output. For normal stars with $(V<$ 18) the goal is to determine $T_{\text {eff }}$ to within $50 \mathrm{~K}$ and both $[\mathrm{M} / \mathrm{H}]$ and $\log g$ to 0.1 dex. The accuracy will be lower for variable stars and for those with bright companions.

\section{GAIA SPECTROSCOPY}

Any star brighter than $I_{c} \sim 16.5$ will have its spectrum observed by an on-board spectroscopic telescope. The spectrograph will record spectra at a resolving power of $R=11,500$ in the wavelength range $848-874 \mathrm{~nm}$. This range was chosen (Munari 1999) because most GAIA stars will be intrinsically red, some of them with a notable interstellar absorption. The interval itself is virtually free from telluric absorption, so it will be possible to supplement GAIA spectra with pre- or post-mission ground based observations. The wavelength interval contains three strong lines of the $\mathrm{Ca}$ II triplet that are present in all dwarfs and giants later than B8. In addition, hot stars show the Paschen series of hydrogen, and spectra of cool stars contain many metallic lines (Fig. 2). No resonant interstellar line is present but the intensity of a diffuse interstellar band at $862 \mathrm{~nm}$ was found to be correlated with reddening (Munari 1999). Peculiar stars are easily detected in this wavelength range (Munari 2002).

The primary goal of spectroscopy is to measure the radial velocity. The accuracy has been studied by observations of GAIA-like spectra (Munari, 


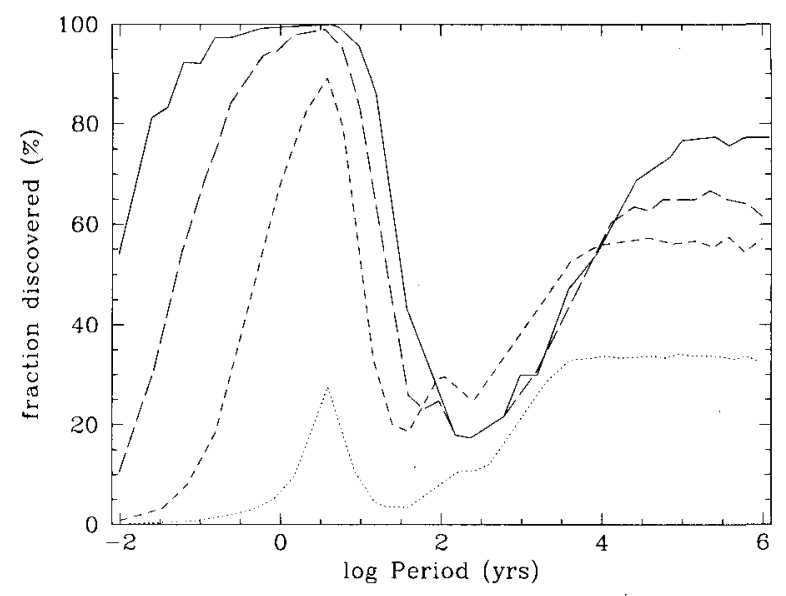

Fig. 3. Fraction of astrometric and resolved binary stars discovered by GAIA as a function of orbital period and for different magnitude ranges: $10<V<12.5$ (solid), $12.5<V<15$ (long dash), $15<V<17.5$ (short dash), $17.5<V<20$ (dotted line). Simulations were done for a distance-limited sample $(d<1 \mathrm{kpc})$. The left maximum marks astrometric systems discovered by a sinusoidal proper motion of the brighter component, the right one is due to systems with resolved components. Adapted from ESA-SCI(2000)4.

Agnolin \& Tomasella 2001a) and simulations (Katz 2000, Zwitter 2002a). Recapitulation (Munari et al. 2003) shows that accuracy of $1 \mathrm{~km} \mathrm{~s}^{-1}$ will be achieved for bright targets (see Table 1). Faint stars will suffer from both a low $\mathrm{S} / \mathrm{N}$ and crowding in the slitless spectrograph focal plane (Zwitter \& Henden 2003; Zwitter 2003a). So spectra of stars fainter than $\mathrm{I}_{\mathrm{c}}=16.5$ are not planned to be recorded.

Comparison with observed (Munari \& Tomasella 1999) and synthetic (Munari \& Castelli 2000; Castelli \& Munari 2001) spectra of standard MKK stars in the GAIA spectral interval could yield other information than radial velocity. The consistency of photometric measurements of temperature, gravity and metallicity could be checked. Abundances of individual elements and rotation velocity (Gomboc 2003) could be measured for the brightest stars. Moreover any peculiarities, including spectroscopic binarity or multiplicity will be readily detected.

All spectral information will be analyzed on the ground. So successive iterations of reduction of each spectrum of a given object could avoid systematic problems, such as unknown multiplicity of the target, that will be discovered during the analysis.

\section{ASTROMETRIC AND RESOLVED BINARIES}

GAIA will be extremely sensitive to non-linear proper motions. So a representative fraction of as-

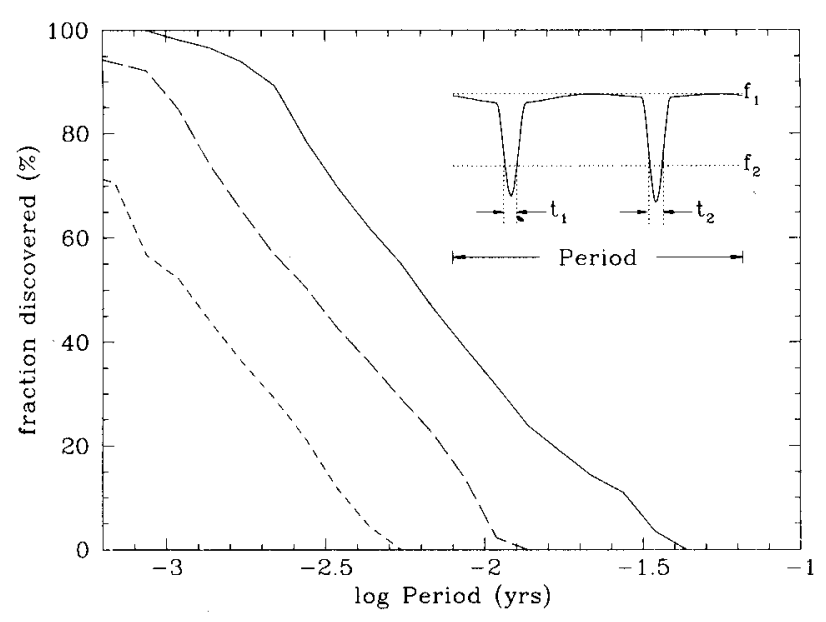

Fig. 4. Fraction of binaries discovered by their photometric variability. The components are assumed to be main sequence stars with a total mass of $2 \mathrm{M}_{\odot}$ and a flat distribution of mass ratios $(0.2<q<1.0)$. A binary gets discovered if its light variation is pronounced enough. If the maximum flux level is $f_{1}$ we assume that the observed flux should be below $f_{2}$ for at least the time $\left(t_{1}+t_{2}\right)=0.08 \times$ Period. The solid curve is for a very accurate photometry where already $f_{2} / f_{1}=0.99$ gets detected. The long dashed one is for $f_{2} / f_{1}=0.95$ and the short dashed one for the least accurate photometry where $f_{2} / f_{1}=0.8$ is needed to beat the noise.

trometric binaries with periods from months to tens of years will be discovered (Fig. 3). GAIA will discover nearly all such binaries brighter than $V=15$ up to a distance of $1 \mathrm{kpc}$. If the orbital period is shorter than a month the binary will be generally too close to discern the sinusoidal component of its proper motion. Periods much longer than the mission lifetime will not be recognized because only a small fraction of the orbit will be observed.

Components in binaries within $1 \mathrm{kpc}$ from the Sun and with periods of a hundred thousand years will be several arc-seconds apart and therefore resolved. Since all six space-velocity coordinates of each component will be measured, the binary nature of the two stars could be recognized.

The same reasoning applies to multiple star systems. We conclude that astrometry can be used to effectively map multiple systems with orbital periods longer than a month over the distances up to a few kpc. For many of the stars with orbital periods up to a few years a complete orbital solution, including masses of the components, will be obtained.

\section{PHOTOMETRIC BINARIES}

Photometric binaries are assumed to be those with an observed photometric variability. They in- 


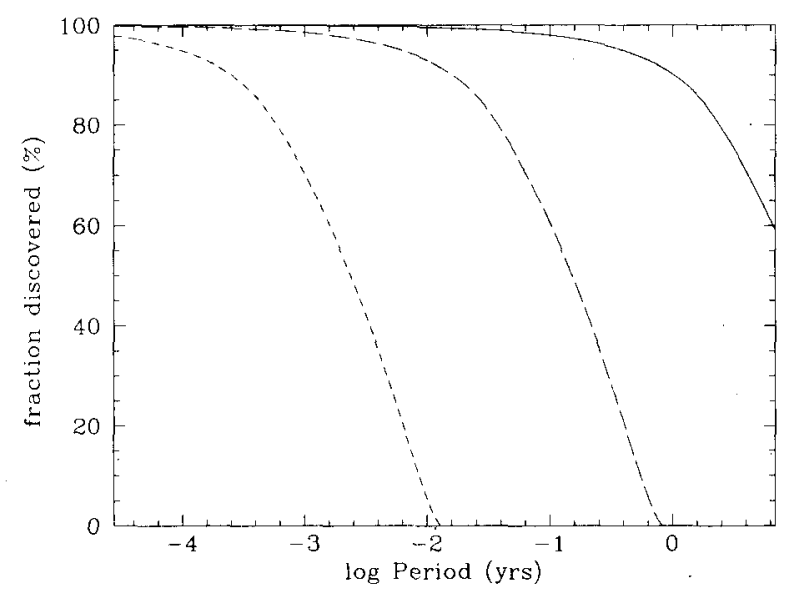

Fig. 5. Fraction of single lined spectroscopic binaries discovered by variation in radial velocity of the more luminous component. The components are assumed to be main sequence stars with a total mass of $2 \mathrm{M}_{\odot}$ and a flat distribution of mass ratios: $0.2<q<1.0$. The curves denote binaries with a $v \sin i$ amplitude of $5 \mathrm{~km} \mathrm{~s}^{-1}$ (solid), $20 \mathrm{~km} \mathrm{~s}^{-1}$ (long dashed) and $80 \mathrm{~km} \cdot \mathrm{s}^{-1}$ (short dashed line). In the case of accurate radial velocity measurements already $5 \mathrm{~km} \mathrm{~s}^{-1}$ variation gets detected and virtually all binaries with periods up to a few years (i.e. the mission lifetime) get discovered. If the measurements are more noisy only large velocity amplitudes, and so binaries with periods of days or less, are recognized.

clude eclipsing binaries, but GAIA photometry will be accurate enough to use the reflection effect and discover some non-eclipsing systems. To illustrate the capabilities of GAIA photometry we performed a series of simulations of binary light curves. The binary modelling code (Wilson 1998) was used via the PHOEBE package (Prša 2003) to simulate a large number of photometric light curves. The components were assumed to be main sequence stars with a total mass of $2 \mathrm{M}_{\odot}$. The mass ratio was uniformly distributed between 0.2 and 1.0 , and the orbital plane was observed at a random orientation. Detailed reflection effects together with proper limb darkening coefficients were taken into account.

The discovery rate of photometric binaries can be characterized by a fraction of the systems that show a certain amplitude of photometric variability. This amplitude should not be reached only at one extreme point in the light curve, i.e. during a short eclipse, because GAIA will observe the system only a limited number of times $(\sim 200$ for middle band and $\sim 82$ for broad band filters). Therefore the binary nature of poorly sampled short eclipses could not be recognized. So we assumed that the flux compared to the maximum flux should stay below a certain

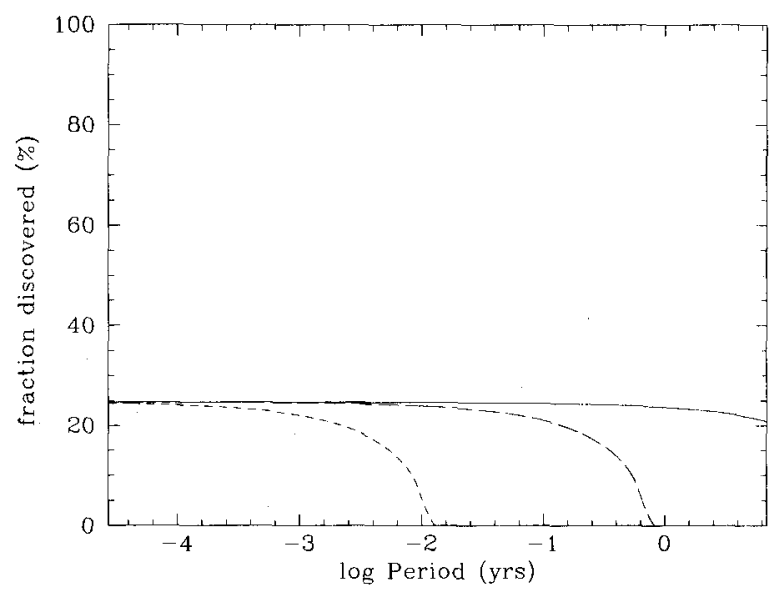

Fig. 6: Fraction of discovered double lined spectroscopic binaries. The components are assumed to be main sequence stars with a total mass of $2 \mathrm{M}_{\odot}$ and a flat distribution of mass ratios $0.2<q<1.0$. We assume that lines from both stars can be measured if the more massive star has at most twice the luminosity of the fainter one. This limits occurrence of double lined systems to $0.8<q<1.0$. The curves denote binaries with a $v \sin i$ amplitude of the more massive component of $5 \mathrm{~km} \mathrm{~s}^{-1}$ (solid), $20 \mathrm{~km} \mathrm{~s}^{-1}$ (long dashed) and $80 \mathrm{~km} \mathrm{~s}^{-1}$ (short dashed line). Interpretation of the required accuracy of radial velocity measurements is similar to Fig. 5 .

level for at least $8 \%$ of the orbital period. Therefore $\sim 16$ middle-band and $\sim 7$ broad-band photometric observations fall into these faint phases. This is enough for a successful determination of the orbital period. The results (Fig. 4) turn out not to depend very much on the length of the considered faint fraction of the light curve. The main parameter is the required amplitude of variation. The solid curve plots fraction of discovered systems if the flux drops $1 \%$ below the maximum value for at least $8 \%$ of the orbital period. The dashed curves are for flux amplitudes of 5 and $20 \%$.

It is clear that photometric variability will most easily discover binaries that are close to contact. For solar-type components these are binaries with orbital periods of a few days or less. The relation of course scales with the size of the binary components and only weakly depends on the total mass. So systems harbouring giants could be discovered at much longer orbital periods. Still, a large luminosity ratio of the components, which is typical for this type of system, will make such discoveries difficult.

Comparing Figs. 3 and 4 shows that photometric variability becomes significant at periods that are just too short for astrometric discovery. GAIA photometry will be of high-enough accuracy (see Ta- 
ble 1) to discover $\approx 50 \%$ of all binaries with such short periods. GAIA's photometric observations will be obtained in several colours simultaneously. So binary light curves could be distinguished from intrinsic variability of a single star, e.g. due to magnetic spots.

\section{SPECTROSCOPIC BINARIES}

Variation of radial velocity is a very efficient way to discover close binaries. In Fig. 5 we plot the situation for a single lined spectroscopic binary. The fraction of discovered systems is plotted for different amplitudes of the light curve of the more luminous component. GAIA will be able to measure single epoch radial velocities with $\approx 20 \mathrm{~km} \mathrm{~s}^{-1}$ precision for stars brighter than $I_{c}=14$. So most systems with orbital periods of a few months or less will be discovered. In the simulation we assumed both stars are main sequence stars with a flat distribution of mass ratios in the range $0.2<q<1.0$. No intrinsic sources of radial velocity variability, like pulsations or giant dark spots on rotating stellar surfaces, were taken into account.

Comparison of Figures 3 and 5 shows that the astrometric and spectroscopic methods nicely complement each other. A significant fraction of systems brighter than $V=15$ could be discovered this way.

Discovery of double lined eclipsing binaries is of utmost importance (e.g. Milone 2003), since only for such systems the mass ratio and eventually the masses themselves could be accurately determined. In Fig 6 we assumed the same conditions as for the single lined binaries, but double lined systems were assumed to have the mass ratio in the range $0.8<q<1.0$. This means that the fainter of the main sequence components still contributes a third of the total light, so its lines can be easily measured. The range of preferred orbital periods is the same as in the single lined case. Assumption on the intrinsic flat distribution of mass ratios in the range $0.2<q<1.0$ implies that at most a quarter of the systems satisfy the additional condition $q>0.8$.

\section{RECOVERY OF PHYSICAL PARAMETERS}

So far we have discussed methods to discover binary and multiple stars. Clearly one wants to know also what would be the accuracy of physical parameters recovered from their analysis. It is sometimes argued that the main role of GAIA is to discover interesting systems and that ground-based follow-up observations can be used for detailed analysis. While this may be true for a few of the most interesting systems it cannot be a general way to proceed. The
TABLE 2

DISTANCE DETERMINATION FOR ECLIPSING DOUBLE-LINED BINARY STARS

\begin{tabular}{lrlll}
\hline System & \multicolumn{4}{c}{ MEASURED DISTANCE (pc) } \\
Designation & \multicolumn{4}{c}{$\begin{array}{c}\text { Hipparcos parallax } \\
\text { min }\end{array}$} \\
& aver. max & \\
\hline V505 Per & 62 & 66 & 70 & $59 \pm 4$ \\
V781 Tau & 73 & 81 & 91 & $81 \pm 1$ \\
UV Leo & 83 & 91 & 103 & $92 \pm 6$ \\
V570 Per & 103 & 117 & 131 & $108 \pm 6$ \\
V432 Aur & 100 & 119 & 146 & $124 \pm 10$ \\
UW LMi & 114 & 129 & 150 & $100 \pm 7$ \\
GK Dra & 246 & 297 & 373 & $313 \pm 14$ \\
CN Lyn & 233 & 362 & 813 & $285 \pm 32$ \\
OO Peg & 304 & 445 & 840 & $295 \pm 17$ \\
\hline
\end{tabular}

${ }^{a}$ From Munari et al. (2001b), Zwitter et al. (2003), Marrese et al. (2004).

numbers of discovered binary and multiple systems will be huge. Figs. 3-5 imply that a significant fraction of binary stars will be discovered at any orbital period. The follow up campaigns will be too time consuming so one should rely on GAIA data alone. A unique feature of GAIA is that any star is observed hundreds of times during the mission. The light curves are therefore reasonably well sampled (e.g. Zwitter 2003a).

A number of studies (Munari et al. 2001b, Zwitter et al. 2003, Marrese et al. 2004) estimated the accuracy of values of physical parameters recovered from GAIA-like observations of eclipsing doublelined spectroscopic binary stars. Hipparcos/Tycho photometry was used as an approximation of GAIA photometry, and the Asiago 1.82-m telescope contributed echelle observations in the wavelength range of the GAIA spectrograph. Altogether $\sim 20$ systems with solar-type components were observed with results on half of them already published. It turns out that masses of individual system components can be obtained at an accuracy of $1-2 \%$. Similar is also the accuracy of other parameters, except for individual stellar radii, which suffer from uneven eclipse sampling. Modelling of the total system luminosity yields also the distance. The values (Table 2 ) are consistent with Hipparcos parallaxes, but generally more accurate. We note that these results are based on a rather noisy Hipparcos photometry, but the precision and number of photometric bands of GAIA will be much higher. The astrometric precision of GAIA will be $\sim 100$-times better than that 
of Hipparcos. But even so some of the distances to the most luminous and remote binary stars will be better determined by system modelling than by astrometry. Niarchos \& Manimanis (2003) discussed the accuracy of recovered physical parameters for near-contact faint systems without spectroscopic information.

GAIA data will be used to solve large numbers of binary and multiple systems containing solar and sub-solar stars. which generally lack accurate solutions in the literature (Andersen 1991). Their absolute placement on the H-R diagram and their exactly equal ages will allow for extensive testing and improvement of the stellar evolution isochrones. It will also close the loop between distances from astrometric parallax and eclipsing binary analysis (Wilson 2003). Some of the stars in binaries will be variable, e.g. of a $\delta$-Scuti type (see Dallaporta et al. 2002, Siviero et al. 2004). Direct determination of their masses and radii from binary system analysis will improve their physical interpretation.

\section{PROJECT RAVE}

The launch of GAIA is planned for the end of the decade. Radial velocities of stars are however a rare commodity (see e.g. Munari et el. 2003), so earlier results are desired. This is the purpose of the RAdial Velocity Experiment (RAVE), an international collaboration led by M. Steinmetz which is starting pilot study observations already in April 2003.

The UK Schmidt telescope at AAO equipped with a fibre-optic spectrograph will be used to obtain spectra in the same wavelength domain (848$874 \mathrm{~nm})$ as GAIA. In the pilot study (2003-2005) about 100,000 stars will be observed, while the main study (from 2006) will survey $\sim 35$ million stars in a magnitude limited $(V=16)$ survey of all the sky accessible from AAO, observing $\sim 5$ million stars per year (or more if an additional telescope in the northern hemisphere could be used).

The primary goal of the RAVE experiment is to measure a large sample of radial velocities. These can be used to address several galactic kinematic issues (Steinmetz 2003).

The pilot study uses the existing hardware of the $6 \mathrm{dF}$ survey to secure spectra at a resolving power of 8500 during bright unscheduled time. The 6-degree FOV is sampled with 150 fibres. Each field of $\sim 130$ stars is exposed for 1 hour. For the main study a new fibre-optic spectrograph will be built, based on the Echidna-style design developed at AAO. It consists of a 2250-spine fibre array that covers the full $40 \mathrm{deg}^{2}$ telescope field of view. Fibres will feed a spectro- graph operating at a resolving power $\sim 10,000$ in the GAIA wavelength range.

The RAVE experiment collects spectra in a way different from GAIA. The latter will scan across any star $\sim 100$-times obtaining a low $\mathrm{S} / \mathrm{N}$ spectrum with an effective exposure time of 100 seconds on each scan. RAVE's main study will obtain a pointed 30 min exposure on each field. A small fraction of stars $(\leq 10 \%)$ will be observed more than once. This means that spectra collected by RAVE will have a good $\mathrm{S} / \mathrm{N}$ ratio, but will lack any time variability information. The expected errors on radial velocity measurements for RAVE are $\leq 2 \mathrm{~km} \mathrm{~s}^{-1}$.

The different observing mode has implications for the discovery and analysis of binary and multiple stars. Single epoch observations could discover only double-lined binaries. Fig. 6 shows that $\sim 10 \%$ of all binaries observed at random orbital phases and with orbital periods up to 10 yrs could be discovered this way. Occasional repeated observations of the same target will have the capacity to discover a single-lined system. High accuracy of radial velocity measurements implies that most of the single-lined spectroscopic binaries with orbital periods similar to, or shorter than, the time span of observations could be discovered.

Discovery of a binary is not enough to assess its physical parameters. GAIA multi-epoch spectroscopy and photometry will provide good epoch information that will generally yield the orbital period and in the lucky cases also a complete set of physical parameters. The spectra obtained by RAVE will generally not have such a capacity. The velocity separation of the two components is statistically linked to the orbital period, and the spectra themselves will reveal the spectral type and metallicity of either component. But a complete analysis will require dedicated follow-up observations. Still the RAVE mission is of extreme importance for binary studies: contrary to photometric surveys it can discover the vast majority of binaries which are not eclipsing. It will provide a great statistical sounding of the binary and multiple star population 10 years ahead of the more complete and detailed GAIA survey.

\section{CONCLUSIONS}

The GAIA mission and RAVE experiment are shifting the focus of binary and multiple star research. The emphasis moves from a dedicated study of a given system to a standardized analysis of a huge sample of such objects. It was estimated (Zwitter 2002b) that GAIA may discover $\sim 7 \times 10^{6}$ eclipsing 
binaries. At least $\sim 10^{4}$ of these will be double-lined and brighter than $V=15$, permitting a reasonable quality determination of their physical parameters. GAIA data will allow for a direct sampling of properties of multiple stars at any orbital period, from minutes to millions of years. The coeval nature of the components in such systems will allow unprecedented tests of evolutionary theories and formation scenarios. The accuracy of distances obtained from analysis of binary systems located at the outskirts of the Galaxy or beyond will rival or supersede those obtained by astrometric measurements. The role of the RAVE experiment will be to discover a large number of the so far unknown binary and multiple star systems and to make a statistical analysis of their properties. Still the joy of making a detailed analysis of an individual binary will not be lost. GAIA and RAVE will merely point to the really interesting cases that justify such a detailed attention.

Generous allocation of observing time with the Asiago telescopes has been vital to this project. The support of the Slovenian Ministry for Education, Science and Sports is acknowledged.

\section{REFERENCES}

Andersen, J. 1991, A\&A Review, 3, 91

Bienaymé, O., \& Turon, C. 2002, eds., GAIA: a European Space Project, (Les Ulis: EDP Sciences), EAS Pub. Ser., vol. 2

Castelli, F., \& Munari, U. 2001, A\&A, 366, 1003

Dallaporta, S., Tomov T., Zwitter, T., \& Munari, U. 2002, IBVS, 5312

Gilmore, G., Perryman, M. A., Lindegren, L., Favata, F., Hog, E., Lattanzi, M., Luri, X., Mignard, F., Röser, S., \& de Zeeuw, P. T. 1998, in SPIE Conf., 3350, Astronomical Interferometry, ed. R. D. Reasenberg, 541

Gomboc, A. 2003, in ASP Conf. Ser., 298, GAIA Spectroscopy, Science and Technology (San Francisco: ASP), ed. U. Munari, 285

Jordi, C., Babusiaux, C., Katz, D., Portell, J., \& Arenou, F. 2003, UB-SWG-012, http://gaia.am.ub.es/ SWG/Documents/UB.SWG_012.pdf

Katz, D. 2000, PhD Thesis, Univ. Paris, 7

Maceroni, C. 2004, this volume

Marrese, P. M., Munari, U., Siviero, A., Milone, E. F., Zwitter, T., Tomov, T., Boschi, F., \& Boeche, C. 2004, A\&A, 413, 635

Milone, E. F. 2003, in ASP Conf. Ser., 298, GAIA Spectroscopy, Science and Technology, ed. U. Munari (San Francisco: ASP), 303
Munari, U. 1999, Baltic Astron., 8, 73

Munari, U., \& Tomasella, L. 1999, A\&AS, 137, 521

Munari, U., \& Castelli, F. 2000, A\&AS, 141, 141

Munari, U., Agnolin, P., \& Tomasella, L. 2001a, Baltic Astron., 10, 613

Munari, U., Tomov, T., Zwitter, T., Milone, E. F., Kallrath, J., Marrese, P. M., Boschi, F:, Prša, A., Tomasella, L., \& Moro, D. 2001b. A\&A, 378, 477

Munari, U. 2002, in ASP Conf. Ser., 279, Exotic Stars as Challenges to Evolution, eds. Tout, C. A. \& Van Hamme, W. (San Francisco: ASP), 25

Munari, U. 2003, ed., ASP Conf. Ser., 298, GAIA Spectroscopy, Science and Technology

Munari, U., Zwitter, T., Katz, D., \& Cropper M. 2003, in ASP Conf. Ser., 298, GAIA Spectroscopy, Science and Technology, ed. U. Munari (San Francisco: ASP), 275

Niarchos, P. G., \& Manimanis, V. N. 2003, A\&A, 405, 263

Perryman, M. A. C., de Boer, K. S., Gilmore, G., Hog, E., Lattanzi, M. G., Lindegren, L., Luri, X., Mignard, F., Pace, O., \& de Zeeuw, P. T. 2001, A\&A, 369, 339

Prša, A. 2003, in ASP Conf. Ser., 298, GAIA Spectroscopy, Science and Technology, ed. U. Munari (San Francisco: ASP), 457

Siviero, A., Munari, U., Sordo, R., Dallaporta, S., Zwitter, T., Marrese, P. M., \& Milone, E. F. 2004, A\&A, accepted, astro-ph/0309691

Steinmetz, M. 2003, in ASP Conf. Ser., 298, GAIA Spectroscopy, Science and Technology, ed. U. Munari (San Francisco: ASP), 381

Straižys, V. 1999, ed., GAIA, Baltic Astron., 8, 1

Vansevičius, V., Kučinskas, A., \& Sudžius, J. 2002, eds., Kluwer, Census of the Galaxy: Challenges for Photometry and Spectrometry with GAIA

Wilson, R. E. 1998, Computing Binary Star Observables, Univ. of Florida Astronomy Dept.

Wilson, R. E. 2003, in ASP Conf. Ser., 298, GAIA Spectroscopy, Science and Technology, ed. U. Munari (San Francisco: ASP), 313

Zwitter, T. 2002a, A\&A, 386, 748

Zwitter, T. 2002b, in ASP Conf. Ser., 279, Exotic Stars as Challenges to Evolution, eds. C. A. Tout \& W. Van Hamme (San Francisco: ASP), 31

Zwitter, T. 2003a, in ASP Conf. Ser., 298, GAIA Spectroscopy, Science and Technology, ed. U. Munari (San Francisco: ASP), 329

Zwitter, T. 2003b, in ASP Conf. Ser., 298, GAIA Spectroscopy, Science and Technology, ed. U. Munari (San Francisco: ASP), 493

Zwitter, T., \& Henden A. 2003, in ASP Conf. Ser., 298, GAIA Spectroscopy, Science and Technology, ed. U. Munari (San Francisco: ASP), 489

Zwitter, T., Munari, U., Marrese, P. M., Přsa, A., Milone, E. F., Boschi, F., Tomov, T., \& Siviero, A. 2003, A\&A, 404, 333 